\section{nephron}

Practice
Nephron 2017;136:281-286

DOI: $10.1159 / 000450618$
Received: March 21, 2016

Accepted after revision: September 5, 2016

Published online: September 20, 2016

\title{
Should We Still Believe in Randomized Controlled Trials in Nephrology?
}

\author{
Monica Cortinovis ${ }^{a}$ Norberto Perico ${ }^{a}$ Giuseppe Remuzzi ${ }^{a-c}$ \\ ${ }^{a}$ Clinical Research Center for Rare Diseases 'Aldo and Cele Daccò', IRCCS - Istituto di Ricerche Farmacologiche Mario \\ Negri, and ' ${ }^{\text {Unit }}$ of Nephrology and Dialysis, Azienda Socio-Sanitaria Territoriale Papa Giovanni XXIII, Bergamo, and \\ 'Department of Biomedical and Clinical Sciences 'L. Sacco', University of Milan, Milan, Italy
}

\section{Key Words}

Nephrology · Renal diseases · Randomized

double-blind trial

\begin{abstract}
The randomized controlled trial (RCT) is the cornerstone upon which clinical decision-making is based. Pivotal RCTs in the nephrology area efficiently demonstrated the renoprotective effects of treatment with renin-angiotensin system inhibitors in patients with diabetic and non-diabetic proteinuric nephropathies. However, there is concern about the increasing cost, complexity and duration of clinical studies. Moreover, recent large RCTs addressing key issues for patients with renal disease failed to achieve definitive conclusions mainly due to critical flaws in the investigational strategies, including the adoption of excessive/fixed doses of the study medications, inappropriate use of the placebocontrolled design, enrollment of low-risk individuals, poor reporting of adverse events or unreliable evaluation of renal function. The information now available on the biases that characterize the current RCTs should serve as a tool to rethink the design, patient selection and implementation of future RCTs in nephrology.

(c) 2016 S. Karger AG, Basel
\end{abstract}

Contribution from the CME Course of the DIABESITY Working Group of the ERA-EDTA, Bergamo, December 4-5, 2015.

\section{KARGER}

๑๑ 2016 S. Karger AG, Basel

E-Mail karger@karger.com

www.karger.com/nef
Chronic kidney disease (CKD) is increasingly recognized as a major public health problem worldwide. In the past 20 years, age-standardized death rates from CKD increased by $15 \%$, whereas rates for other non-communicable diseases declined, including major vascular diseases, pulmonary disorders, most forms of cancer and liver cirrhosis [1]. Despite this, it has been claimed that in the past 20 years, kidney research has been associated with a lower number of randomized controlled trials (RCTs) as compared to other medical specialties [2]. This is not true, however, if one corrects for the number of patients affected and the dedicated investigators. Nevertheless, the reputation of randomized trials has suffered owing to concern about increasing complexity, study design, expense, time required to recruit study participants, as well as inadequate representativeness of enrollees for the general patient population [3]. Clearly, this dismal state of affairs has created a challenge for nephrologists to improve clinical decision-making upon the basis of highquality RCTs.

\section{Successful RCTs in Nephrology}

Pioneering large, simple RCTs were designed to address straightforward questions about the value of novel therapeutic strategies on important health outcomes, such as renal disease progression to end-stage renal dis-

Prof. Giuseppe Remuzzi

Clinical Research Center for Rare Diseases 'Aldo and Cele Daccò

IRCCS - Istituto di Ricerche Farmacologiche 'Mario Negri'

Via GB Camozzi 3, IT-24020 Ranica, Bergamo (Italy)

E-Mail giuseppe.remuzzi@ marionegri.it 
ease (ESRD) and mortality. Studies of this kind were useful to demonstrate the renoprotective effects of renin-angiotensin system (RAS) inhibition in patients with diabetic nephropathy. Indeed, Bjorck et al. [4] reported that, at comparable blood pressure, the angiotensin-converting enzyme inhibitor (ACEi) enalapril reduced the rate of glomerular filtration rate (GFR) decline more than the treatment with a beta blocker in type 1 diabetic patients with overt nephropathy. In 1993, the seminal Collaborative Study [5] found less progression to the combined endpoint of doubling serum creatinine, ESRD or death while on the ACEi captopril compared to placebo in 409 patients with type 1 diabetes and overt nephropathy. Almost 10 years later, the Reduction of Endpoints in noninsulin-dependent diabetes mellitus with the Angiotensin II Antagonist Losartan (RENAAL) trial [6] and the Irbesartan in Diabetic Nephropathy Trial [7] showed that angiotensin-receptor blocker (ARB) treatment, as compared with placebo, reduced the incidence of a composite endpoint of doubling of serum creatinine concentrations, ESRD, or death by 16 and 19\%, respectively, in 2 large cohorts of patients with type 2 diabetes and overt nephropathy. Renoprotection was associated with a significant reduction in urinary protein excretion; this finding remained significant after adjustment for the small differences in blood pressure control between treatment groups. Independent of treatment allocation, both trials showed that an early reduction in urinary protein excretion was associated with slower renal function loss and reduced cardiovascular mortality in the long-term [8]. The first observation that RAS inhibition can prevent the development of overt proteinuria was provided by the Irbesartan Microalbuminuria Type 2 Diabetes in Hypertensive Patients (IRMA)-2 study, an RCT involving 590 hypertensive patients with type 2 diabetes and microalbuminuria [9]. A median of 2-year treatment with full dose $(300 \mathrm{mg} /$ day) irbesartan was associated with a threefold reduction in the incidence of macroalbuminuria compared with placebo [9]. Similar studies have also efficiently demonstrated the renoprotective effects of ACEi therapy in patients with non-diabetic progressive chronic nephropathies. Indeed, prior to 1995, several small, randomized trials tested ACEi treatment in patients with non-diabetic renal disease, but they did not report uniform results. Possible sources of variability included different methods to measure renal function, different causes and severity of renal disease, use of different ACEi, and small sample sizes. Then, the Angiotensin-Converting-Enzyme Inhibition in Progressive Renal Insufficiency study showed that the ACEi benazepril reduced the risk of serum creatinine doubling compared to placebo in patients with non-diabetic CKD, but a difference in blood pressure between the 2 treatments left open the question of whether the renoprotective effect of the active drug was related to its antiproteinuric property or to better pressure control [10]. Convincing evidence of the renoprotective potential of ACEi therapy was first provided by the triad of Lancet publications generated by the Ramipril Efficacy in Nephropathy study from 1997 to 1999 [11]. At equivalent blood pressure control, the ACEi ramipril reduced the rate of GFR decline and halved the risk of progression to ESRD compared to placebo in patients with non-diabetic proteinuric nephropathies [11]. Ramipriltreated patients also enjoyed a greater decrease in proteinuria that inversely correlated with the rate of GFR decline, indicating that nephroprotection was eventually linked to the reduction of protein traffic through the glomerular capillary barrier [11]. As large-scale trials became an accepted way to evaluate new therapies, the original intent of simplicity was lost while large sizes were maintained, leading to increasingly complex trials. Indeed, between 2002 and 2012, the average number of eligibility criteria in a typical phase 3 protocol dramatically jumped from 31 to 50 and the mean number of study procedures increased from 106 to 167 [12]. The unintended consequence has been to threaten the very existence of RCTs, given the operational complexities and ensuing costs.

\section{Failure of Recent RCTs in Nephrology}

A number of recent RCTs addressing core issues for patients with renal diseases failed to draw firm conclusions because of crucial flaws in the investigational strategies, such as adoption of too high and/or fixed doses of study drugs, inappropriate use of the placebo-controlled design, enrollment of low-risk patients, poor reporting of adverse events or unreliable assessment of renal function.

\section{Inappropriate Selection of Drug Dose}

The adoption of too high and/or fixed doses of investigational compounds can inflate the incidence of adverse events and can lead to the early closure of an RCT for safety reasons, eventually precluding the opportunity to demonstrate clinically relevant treatment effects. This issue has been illustrated by the Veteran Affairs Nephropathy in Diabetes (VA NEPHRON-D) trial that compared double RAS blockade with the ACEi lisinopril plus ARB losartan versus losartan monotherapy on a primary com- 
posite endpoint of changes in estimated GFR (eGFR), ESRD and death in 1,448 patients with type 2 diabetes and overt nephropathy [13]. The study was prematurely terminated because of concerns about a high prevalence of hypotension, hyperkalaemia and acute kidney injury with dual RAS therapy. In fact, these adverse events could have been prevented by avoiding forced ACEi up-titration (up to $40 \mathrm{mg}$ lisinopril daily, in patients with an eGFR as low as $30 \mathrm{ml} / \mathrm{min} / 1.73 \mathrm{~m}^{2}$ ) on top of full-dose losartan. Of note, at study closure dual versus single RAS inhibition had already reduced ESRD events by $34 \%$, a treatment effect never reported before in type 2 diabetes. Risk reduction was associated with a significantly greater decline in proteinuria and approached nominal significance $(\mathrm{p}=$ 0.07 ) over just 2.2 years of follow-up. In the RENAAL study, in a similar cohort of patients with type 2 diabetes, the larger antiproteinuric effect of losartan was associated with a similar (28\%) ESRD reduction compared to placebo. The treatment effect was, however, not still appreciable at 2.2 years, but became statistically significant over the planned 3.2 years of follow-up [6]. These data strongly suggest that even in the VA NEPHRON-D trial, ESRD events could have been significantly reduced over the initially scheduled 5-year study period. Of course, dual blockade therapy requires diligence by clinicians to ensure that RAS inhibition is held during acute illness, but the benefits are clearly worth the extra effort.

\section{Inappropriate Use of the Placebo-Controlled Design}

Clinical trials of novel medications should not use a placebo control group when proven effective therapies already exist for the condition, preferring active controls. Indeed, superiority trials over placebo may allow drugs into the market that are in fact less effective or safe than those already available, and do not help define the clinical value of new medications with respect to treatments currently in use. In this regard, the Bergamo Nephrologic Diabetes Complication Trial (BENEDICT) was the first large-scale trial designed to assess whether an ACEi could prevent the onset of microalbuminuria in hypertensive but normoalbuminuric patients with type 2 diabetes [14]. Overall, 1,204 patients were randomized to the ACEi trandolapril alone, the calcium-channel blocker verapamil alone, trandolapril plus verapamil or placebo. After 3.6 years of follow-up, trandolapril alone or in combination with verapamil reduced the risk of progression to microalbuminuria from 10.9 to $5.8 \%$ compared to nonACEi therapy, an effect that was observed at comparable blood pressure control [14]. A virtually identical study, the Randomised Olmesartan and Diabetes Microalbu-

Should We Still Believe in RCTs in Nephrology? minuria Prevention (ROADMAP) trial, was published 7 years later with an ARB instead of an ACEi in 4,447 patients with type 2 diabetes and normoalbuminuria. After 3.2 years of follow-up, olmesartan therapy reduced the incidence of microalbuminuria compared to placebo from 9.8 to $8.2 \%$, an effect that was no longer significant when adjusting for blood pressure in the 2 treatment arms [15]. The use of a placebo-controlled design for ROADMAP appeared questionable considering that BENEDICT had already demonstrated a clear benefit of trandolapril in preventing microalbuminuria in a similar patient population. The inclusion of an ACEi arm in ROADMAP could have informed about the renoprotective effects of olmesartan in relation to those of trandolapril. A comparative study would have provided data as well on the adverse event rates associated with these treatments, since indirect comparison of hard outcomes from ROADMAP and BENEDICT indicated a higher risk of cardiovascular mortality with olmesartan than with trandolapril [16].

\section{Post-Treatment Assessment in RCT Design}

RAS blockade has been almost universally considered the key approach for renoprotection in proteinuric diabetic and non-diabetic kidney diseases, mainly via its ability to prevent the development of proteinuria or lowering urinary protein excretion rate in patients already with overt nephropathy. However, at least for the prevention target, most studies do not provide formal evidence that indeed RAS blockade is capable, for example, to prevent the appearance of microalbuminuria in type 2 diabetes $[14,15]$, since post-treatment assessment is not usually included in the RCT design. Instead, if microalbuminuria becomes apparent after stopping RAS blockade, then the conclusion would be that these drugs are antiproteinuric. Therefore, a critical issue in RCT design would be to always include a short post-treatment assessment period that could help to get insights on the mechanism(s) of the potential renoprotection of drugs under investigation, as shown by the sub-study of the IRMA-2 [17] and the Bardoxolone Methyl Treatment: Renal Function in CKD/Type 2 diabetes (BEAM) study [18].

\section{Enrollment of Low-Risk Patients}

Trial enrollment of patients with low risk for outcome events may dilute or even mask any real treatment effect. As an example, a post-hoc analysis of the Ongoing Telmisartan Alone and in combination with Ramipril Global Endpoint Trial (ONTARGET) suggested that

Nephron 2017;136:281-286

DOI: $10.1159 / 000450618$ 
combined therapy with the ACEi ramipril and ARB telmisartan increased the risk of the pre-specified composite endpoint of any dialysis, serum creatinine doubling or death compared with either agent alone in 25,620 patients with atherosclerotic disease and/or diabetes with end-organ damage [19]. However, the excess of adverse renal outcomes on combination therapy was mainly driven by the more frequent need for short-term dialysis, conceivably a treatment-related acute effect on renal hemodynamics that is reversible upon treatment withdrawal [20]. Conversely, the incidence of ESRD was similarly rare in the 3 groups, reflecting the slow rate of renal function loss that, independent of treatment allocation, was close to that observed in the general population as a result of aging and also similar to that reported in patients who have diabetes or hypertension, without proteinuria [21]. Indeed, the slow rate of GFR decline and the lack of any appreciable improvement in renal outcomes following RAS inhibitors therapy can be largely explained by the fact that only $4 \%$ of the study participants had overt proteinuria [20]. This observation highlights that the ONTARGET renal sub-study was actually off target, due to the selective bias of patients with low-risk renal disease progression, given the lack of proteinuria in most of them [20]. In proteinuric nephropathies, proteinuria reduction is one important treatment target [22], and dual RAS blockade is the most efficient way of achieving this target [23]. Thus, the ONTARGET sub-study findings should not be extended to patients with chronic proteinuric nephropathies, in whom proteinuria reduction can delay or even prevent the progression of renal disease towards ESRD.

\section{Poor Reporting of Adverse Events}

Excessive optimism that stemmed from positive preliminary results can cause the importance of troublesome adverse reactions to be overlooked. A paradigmatic example is represented by bardoxolone methyl, an anti-inflammatory agent that was tested as a treatment for diabetic nephropathy in the BEAM study, a phase 2, doubleblind RCT [18]. Bardoxolone methyl significantly increased eGFR compared to placebo, but the trial report tended to underestimate the adverse event profile of the drug, which included worsening proteinuria, massive weight loss, hypomagnesemia, liver function disarray and gastrointestinal effects. These adverse reactions to bardoxolone methyl were not analyzed in detail and the phase 3 Bardoxolone Methyl Evaluation in Patients with Chronic Kidney Disease and Type 2 Diabetes Mellitus: the Occurrence of Renal Events (BEACON) trial was ini- tiated in patients with advanced diabetic nephropathy. Enrollment was completed, but after only 9 months of follow-up, the trial was stopped on the recommendation of its independent data-monitoring committee, which reported a safety imbalance between the bardoxolone and the placebo arms [24]. Adverse events associated with study closure included unintended weight loss, increased proteinuria, and more gastrointestinal symptoms in the bardoxolone group (all already noted in the BEAM study), in addition to higher rates of heart failure and cardiovascular events versus placebo. Of note, later studies conducted in diabetic Zucker rats given the bardoxolone methyl analog RTA 405 reproduced most of the side effects observed in the BEAM and BEACON trials [25], which were, however, attributed to meaningful levels of impurities and/or degradation products in the compound preparation, which could have contributed to the toxicity. Nevertheless, some diabetic rats treated with another bardoxolone methyl analog dh404 (that was free of any potential toxic impurities) but not with vehicle, showed the presence in the kidney of granulomatous and inflammatory process that was suspicious of a pseudotumor [25]. These results were at variance with subsequent study in a similar rat model by investigators from the drug company sponsoring the BEACON trial, who did not find adverse effects of both synthetic bardoxolone analogues [26]. However, it should be pointed out that activation of the nuclear factor erythroid 2-related factor, as it occurs with bardoxolone methyl, in a knockdown model of leptindeficient mice increased insulin resistance and caused anorexia and hepatic steatosis [27], possibly providing an additional warning sign that this drug may be harmful in particular conditions. Nevertheless, besides the adverse events, the major design pitfall in BEACON was the failure to carefully consider what worked well in terms of patient selection in the phase 2 BEAM trial and the use of a fixed maximal dose of bardoxolone methyl instead of a dose up-titration approach. Thus, the valuable lesson is that a meticulous examination of available clinical data and an unbiased analysis of experimental findings would avert the failure of large-scale RCTs.

\section{Unreliable Assessment of Renal Function}

CKD is often characterized by a slow, progressive loss of renal function that may eventually lead to ESRD. GFR estimating equations are widely used in randomized clinical trials testing treatment effects on renal disease progression. These formulas, however, do not allow a rigorous assessment of renal function, and their use may generate misleading information. The importance of this 
point can be appreciated analyzing the results of the A Long-Acting somatostatin on DIsease progression in $\mathrm{Ne}$ phropathy trial due to autosomal dominant polycystic kidney disease (ALADIN); this trial compared the effects of 3-year treatment with Octreotide-LAR or placebo on kidney and cyst growth and renal function decline in patients with autosomal dominant polycystic kidney disease (ADPKD) [28]. At 1 year follow-up, reduction of GFR measured by the plasma clearance of unlabeled iohexol was similar between groups, but subsequent chronic GFR decline from years 1 to 3 was significantly slower with Octreotide-LAR compared to placebo. Intriguingly, when renal function was estimated by the abbreviated Modification of Diet and Renal Disease (aMDRD) equation, no difference in chronic eGFR changes (from years 1 to 3 ) was observed between the Octreotide-LAR and the placebo groups (Piero Ruggenenti, Personal communication). Thus, in the ALADIN trial, the clinically relevant renoprotective effect of Octreotide-LAR would have been missed if GFR had not been measured, but just estimated with equations. In the same vein, the prediction formulas CKD Epidemiology Collaboration and aMDRD unreliably estimated actual GFR values, and failed to predict GFR changes over time in a cohort of adult patients with ADPKD, independent of their kidney function [29]. Similarly, evidence is available that questions the use of any GFR estimation formula to monitor renal disease progression and response to treatment in type 2 diabetics with normo- or microalbuminuria [30]. Therefore, direct measurements of GFR by gold-standard techniques based on the use of suitable exogenous compounds (i.e., inulin, iohexol, iothalamate) would allow a more accurate assess- ment of the potential renoprotective effects of novel drugs, and should be strongly advised to monitor renal function change in RCTs.

\section{Conclusions}

RCTs have become a cornerstone of evidence-based medicine, and therefore have an important impact on clinical decision-making. However, recent trials in the nephrology field achieved inconclusive findings as a result of the inappropriate choice of investigational strategies. Enough information is now available on the biases present in the current RCTs, and should serve as tool to rethink the design, patient selection, implementation and reporting of future RCTs. It should also be appreciated that very large studies do not always represent the best option since they often detect very small, statistically significant differences in outcomes that, however, have little or no clinical relevance. The use of the gold-standard tools, such as measured GFR, to assess treatment effects on renal disease progression would increase the power of statistical analyses, and eventually allow the execution of RCTs with affordable sample size and follow-up duration. Better interactions between academia, pharmaceutical industry and regulatory authorities would be of paramount importance for ensuring the quality, efficacy and safety of drugs in RCTs.

\section{Disclosure Statement}

The authors declare no conflicts of interest.

\section{References}

1 Lozano R, Naghavi M, Foreman K, et al: Global and regional mortality from 235 causes of death for 20 age groups in 1990 and 2010: a systematic analysis for the global burden of disease study 2010. Lancet 2012;380:20952128.

2 Palmer SC, Sciancalepore M, Strippoli GF: Trial quality in nephrology: how are we measuring up? Am J Kidney Dis 2011;58:335337.

3 Malakoff D: Clinical trials and tribulations. Spiraling costs threaten gridlock. Science 2008;322:210-213.

4 Bjorck S, Mulec H, Johnsen SA, et al: Renal protective effect of enalapril in diabetic nephropathy. BMJ 1992;304:339-343.

5 Lewis EJ, Hunsicker LG, Bain RP, et al: The effect of angiotensin-converting-enzyme in- hibition on diabetic nephropathy. The collaborative study group. N Engl J Med 1993;329: 1456-1462.

6 Brenner BM, Cooper ME, de Zeeuw D, et al: Effects of losartan on renal and cardiovascular outcomes in patients with type 2 diabetes and nephropathy. N Engl J Med 2001;345:861869.

7 Lewis EJ, Hunsicker LG, Clarke WR, et al: Renoprotective effect of the angiotensin-receptor antagonist irbesartan in patients with nephropathy due to type 2 diabetes. $\mathrm{N}$ Engl J Med 2001;345:851-860.

8 de Zeeuw D, Remuzzi G, Parving $\mathrm{HH}$, et al: Albuminuria, a therapeutic target for cardiovascular protection in type 2 diabetic patients with nephropathy. Circulation 2004;110:921927.
9 Parving HH, Lehnert $\mathrm{H}$, Brochner-Mortensen J, et al: The effect of irbesartan on the development of diabetic nephropathy in patients with type 2 diabetes. N Engl J Med 2001;345:870878.

10 Maschio G, Alberti D, Janin G, et al: Effect of the angiotensin-converting-enzyme inhibitor benazepril on the progression of chronic renal insufficiency. The angiotensin-convertingenzyme inhibition in progressive renal insufficiency study group. N Engl J Med 1996;334: 939-945.

11 Randomised placebo-controlled trial of effect of ramipril on decline in glomerular filtration rate and risk of terminal renal failure in proteinuric, non-diabetic nephropathy. The GISEN group (Gruppo Italiano di Studi Epidemiologici in Nefrologia). Lancet 1997;349:1857-1863.
Should We Still Believe in RCTs in Nephrology?
Nephron 2017;136:281-286 DOI: $10.1159 / 000450618$ 
12 Getz K: Improving protocol design feasibility to drive drug development economics and performance. Int J Environ Res Public Health 2014;11:5069-5080.

13 Fried LF, Emanuele N, Zhang JH, et al: Combined angiotensin inhibition for the treatment of diabetic nephropathy. N Engl J Med 2013;369:1892-1903.

14 Ruggenenti P, Fassi A, Ilieva AP, et al: Preventing microalbuminuria in type 2 diabetes. N Engl J Med 2004;351:1941-1951.

15 Haller H, Ito S, Izzo JL Jr, et al: Olmesartan for the delay or prevention of microalbuminuria in type 2 diabetes. N Engl J Med 2011;364: 907-917.

16 Ruggenenti P, Cravedi P, Remuzzi G: Mechanisms and treatment of CKD. J Am Soc Nephrol 2012;23:1917-1928.

17 Andersen S, Brochner-Mortensen J, Parving $\mathrm{HH}$; Irbesartan in Patients with Type 2 Diabetes and Microalbuminuria Study Group: Kidney function during and after withdrawal of long-term irbesartan treatment in patients with type 2 diabetes and microalbuminuria. Diabetes Care 2003;26:3296-3302.

18 Pergola PE, Raskin P, Toto RD, et al: Bardoxolone methyl and kidney function in CKD with type 2 diabetes. N Engl J Med 2011;365: 327-336.
19 Mann JF, Schmieder RE, McQueen M, et al: Renal outcomes with telmisartan, ramipril, or both, in people at high vascular risk (the ONTARGET study): a multicentre, randomised, double-blind, controlled trial. Lancet 2008;372:547-553.

20 Ruggenenti P, Remuzzi G: Proteinuria: is the ONTARGET renal substudy actually off target? Nat Rev Nephrol 2009;5:436-437.

21 Casas JP, Chua W, Loukogeorgakis S, et al: Effect of inhibitors of the renin-angiotensin system and other antihypertensive drugs on renal outcomes: systematic review and metaanalysis. Lancet 2005;366:2026-2033.

22 Ruggenenti P, Perna A, Remuzzi G: Retarding progression of chronic renal disease: the neglected issue of residual proteinuria. Kidney Int 2003;63:2254-2261.

23 Ruggenenti P, Perticucci E, Cravedi P, et al: Role of remission clinics in the longitudinal treatment of CKD. J Am Soc Nephrol 2008; 19:1213-1224.

24 de Zeeuw D, Akizawa T, Audhya P, et al: Bardoxolone methyl in type 2 diabetes and stage 4 chronic kidney disease. N Engl J Med 2013; 369:2492-2503.

25 Zoja C, Corna D, Nava V, et al: Analogs of bardoxolone methyl worsen diabetic nephropathy in rats with additional adverse ef- fects. Am J Physiol Renal Physiol 2013;304: F808-F819.

26 Chin M, Lee CY, Chuang JC, et al: Bardoxolone methyl analogs RTA 405 and dh404 are well tolerated and exhibit efficacy in rodent models of type 2 diabetes and obesity. Am J Physiol Renal Physiol 2013;304:F1438F1446.

$27 \mathrm{Xu} \mathrm{J,} \mathrm{Kulkarni} \mathrm{SR,} \mathrm{Donepudi} \mathrm{AC,} \mathrm{et} \mathrm{al:} \mathrm{En-}$ hanced Nrf2 activity worsens insulin resistance, impairs lipid accumulation in adipose tissue, and increases hepatic steatosis in leptin-deficient mice. Diabetes 2012;61: 3208-3218.

28 Caroli A, Perico N, Perna A, et al: Effect of longacting somatostatin analogue on kidney and cyst growth in autosomal dominant polycystic kidney disease (ALADIN): a randomised, placebo-controlled, multicentre trial. Lancet 2013;382:1485-1495.

29 Ruggenenti P, Gaspari F, Cannata A, et al: Measuring and estimating GFR and treatment effect in ADPKD patients: results and implications of a longitudinal cohort study. PLoS One 2012;7:e32533.

30 Gaspari F, Ruggenenti P, Porrini E, et al: The GFR and GFR decline cannot be accurately estimated in type 2 diabetics. Kidney Int 2013; 84:164-173. 